\title{
An IoT, Virtual Machines and Cloud Computing-based Framework for an Optimal Management of Healthcare Data Collected from a Smart Environment. A Case Study: RO-Smart Ageing Project
}

\author{
Dragoș Nicolae NICOLAU, Adriana ALEXANDRU, Marilena IANCULESCU \\ National Institute for Research and Development in Informatics \\ dragos.nicolau@ici.ro, adriana.alexandru@ici.ro, marilena.ianculescu@ici.ro
}

\begin{abstract}
Globally, the population is ageing and the number of older people is increasing dramatically. These are challenges for healthcare systems because an increased need for medical assistance is related to ageing. The availability of health-related data and information is a main issue for assessing the health status of seniors, having an important impact on their quality of life. Due to Internet of Things (IoT), cloud computing, smart environment, and virtual machines, the traditional healthcare approaches are replaced by up-to-date (remote) health monitoring systems in smart environments. The paper presents the opportunities provided by such solutions for an appropriate management of older people health related information. RO-SmartAgeing, a remote health monitoring system based on IoT, is presented as a case study with the intention of revealing the rise of the efficiency and the decease of the response time in seniors remote monitoring systems.
\end{abstract}

Keywords: Data Optimized Management, Ageing Population, Internet of Things, Virtual Machine, Elderly

1 Introduction

The total ageing population is increasing constantly due to the decline of fertility (caused by increased level of education, urbanization, access to contraceptive methods or shifting from the common family purposes to individual needs), improvements of healthcare services, policies and health literacy, advancements in health domain and continuously emerging technology. In the same time, the expenditure for elderly healthcare is increasing too.

According to Eurostat's latest set of population, 'the EU-28's population is projected to increase to a 528.6 million around 2050 and thereafter gradually decline to 518.8 million by 2080. [1]. By 2050, the number of people aged 65 or older (considered as elderly people) is expected to be about 1.5 billion, representing 16 percent of the world's population. [2].

The current ageing society comes both with challenges and opportunities. Among the main challenges are: a decreased labour force participation, higher expenditures of healthcare and social systems, lack of ageingspecialized caretakers, appropriate agefriendly environments etc. Nevertheless, a diversity of opportunities come along with ageing: implementing new models and patterns for an independent, active and healthy living, personalized medicine and therapeutically protocols, broadly developing smart cities, communities and technologies, citizens' empowerment.

Older population implies a demand for transforming the healthcare services from reactive to proactive ones, with an agefriendly, elderly patient-centred and personalized approach, focused towards prevention and avoiding hospitalization and institutionalization in nursing-homes as much as it is beneficial for the senior and its family. Smart environments represent one important mean to provide a dignified and qualitative life of the elderly. A smart environment should be able to ensure a safe, personalized and secure home in which the daily activities and health status parameters of the senior, together with the ambient parameters can nonintrusively be monitored in order to set a triggered alarm in case of the occurrence of a (potential) harmful event or for a customized healthcare management.

In this respect, the remote monitoring can be operated at several scales: at the individual 
level (aiming to provide e-services for supporting an independent and healthy life of the senior under a customized supervision of health specialists), at the group level (incorporating persons with common interests and goals in health monitoring applications) and at the community level (aiming to use collective data analytics gathered from a very large number of people in their benefit) [3].

The Internet of Things (IoT) technology provides the capabilities of gathering and sharing a huge diversity of multiple-source data, increased level of interconnectivity, being one of the main bases for remote monitoring. Wearable devices and smart technology have the potential to raise the quality of life by providing e-services able to support the preserving and even the recovery of the independence and health of people.

Virtualization can be defined as the capability of simulating or emulating an object or resource in software for making it identical to the corresponding physical object; i.e. the abstracting makes the software look and behave like hardware, with appropriate benefits in flexibility, cost, scalability, reliability, performance, and in a broad range of applications [4].

Cloud computing technology represents a main driver in relation with the IoT inside computation developing, energy efficiency, or service implementation [5]. Cloud computing facilitates the sharing of resources by different users (they are tenants with respect to the Cloud Unit), providing them the impression of a virtually infinite computing infrastructure. The employment of virtual machines (VM) in a cloud environment ensures an improved scalability for dynamically set up the growing resource need [6]. Multiple VM configurations needs to be intelligently allocated in order to manage optimally the data collected from a smart environment.

This paper aims to present a proposed conceptual solution for an optimized management of healthcare data, with a focus on the aggregation among IoT, virtual machines and cloud computing capabilities.

The specific technologies associated to a smart environment (such as Internet of
Things, Cloud computing, Virtual Machines, etc.) are introduced in chapter 2 of the paper. A proposed conceptual solution for an optimized management of healthcare data, with a focus on the aggregation among IoT, virtual machines and cloud computing capabilities is presented in chapter 3 . The solution is intended to be developed inside the ongoing RO-SmartAgeing project which is briefly reviewed.

\section{Customised Smart Services for Senior Patients Generated By Smart Environments}

\subsection{A Growing, Demanding Ageing Population}

Population ageing is the main social transformation all over the world. By 2050, the number of people aged 65 or older is expected to be about 1.5 billion, representing 16 percent of the world's population. [7].

In many advanced countries, various problems caused by this fact have appeared, such as economic problems related to reduced productivity or increased medical and care costs, and social welfare problems related to old people care and social security. Besides, the social participation, leisure and lifelong learning needs of the seniors would have been taken into account. Thus, due to the global ageing, there are growing demands for material and financial resources in health and social systems. The provision of smart health services would enable older adults to be permanently in touch with the medical professionals or caregivers satisfying the growing accountability to their own health.

\subsection{Smart Environments Customised For Senior Patients}

Taking into consideration the global aging of population, it has become necessary to clearly identify the specific seniors' health problems, needs, living conditions and life experiences, and to provide real time communication between health care professionals and patients.

\section{Distinctive performances/features}

Challenges generated by the global ageing of population enforce distinctive performances 
to be offered in healthcare focused on senior patients. Here are some of the most important ones: Emergence and rapid development of intelligent products aimed at improving the life quality of the elderly users, caused by the development of science and technology; Ability to achieve better access to care, increased treatment options, and the creation of new opportunities for personalized and patient-centred medical acts; Provision of new ways to deliver personalized health care and health care services; Migration to the patient-centred healthcare by monitoring and integrating patient's medical data collected in and out of a clinical environment in treatment protocols, clinical research studies, remote monitoring programs, and preventive actions and measures; Continuing surveillance of the physical signs of older adults with worse agerelated health status; Enabling users to receive immediate feedback on their physical conditions anywhere and anytime due to the advancement of health information technologies; Reduction of physical check-up cost and decreasing the risk of hospital admission by using wearable systems at home; Facilitating the healthcare delivery process and mitigating the social burden by ubiquitous usage of smart wearable systems for older adults; Providing cost-efficient adapted (and increasingly complex) care services in senior residences.

\section{Specific technologies}

The request of the ageing population for healthcare services and assistance is increasing. The convergence between innovative digital devices and technologies and health care services provides real-time gathering of medical / environmental data and information, and the availability of massive amounts of patient-related data.

Older people have increasingly adopted digital technologies, facing different types of physical, psychological and social challenges. The acceptance of digital technologies in everyday life is influenced by the variation of degenerative disorders or technological literacy and background [8].

The Internet of Things (IoT) is a recent paradigm related to the interconnection of a huge amount of heterogeneous objects (things) through the Internet. This is granted by the latest technological advances in smart sensors, communication, and Internet protocols. In healthcare, IoT reduces the risks of human errors during data acquisition by using the automatic medical data collection method. This leads to the improvement of the quality of the diagnosis and to the reduction of the risk of human errors during gathering or transmission of false information, which could have a negative impact on the patients' health. The application of IoT in healthcare is divided into [9]: Clinical care: sensors are used to collect physiological information, which is analysed for identifying emergency condition for risky patients, stored on the cloud and sent via Internet to caregivers (patient's family members, nurse) for further analysis; Remote monitoring: is useful for elderly, children or people with chronic illnesses (such as cardiopulmonary disease, Asthma, and Heart failure), which are located far away from medical care facilities. It help caregivers to have real-time pre-diagnosis and earlier intervention before things go wrong; Context-awareness: provides the ability to find the patient's condition and the environment where the patient was located by using different types of sensors to acquire various information about the patient's physical condition (such as his walking, running, sleeping etc.). Such information is helpful in emergency cases for patient' location and for being aware of the type of recommended emergency intervention.

IoT computing aims at automated extraction of data from Internet-connected things and at controlling them in a more informed fashion. [10].

Wireless Sensor Networks (WSNs) is another related technology. Such a network consists of interconnected sensors, each equipped with a processor and a flash memory, and can be interfaced to a computer via a gateway [11].

Smart wearable health monitoring technologies are used to detect deteriorations in cognitive ability, mobility, and psychosocial functioning of older adults [12]. Physical condition data are transmitted, 
through wireless sensor networks, to a medical centre for providing real-time healthcare to patients.

A smart wearable system (supported by technologies, such as wireless sensor networks and electronic care monitoring devices) can be implemented by using wearable sensors (smart clothes, implantable devices, skin devices, and other wearable gadgets), actuators, and smart devices for health evaluation and decision support. Such systems mainly work in monitoring vital signs, body movement, location, and fall prevention [13], [14].

The smart wearable systems for health monitoring include smart clothes, implantable devices, skin devices, and other wearable gadgets [15]. For example, equipped with WSN, a smart wearable device can be used to obtain vital parameters data (e.g. ECG), to monitor current activity with a three-axis accelerometer sensor, and to detect old patient position and real-time human falls.

The paradigm of Cloud computing is supplying everything as a service, provided that services are virtualized, pooled, shared, fit up and quickly disposable with minimum management burden. From the users perspective, these services can be accessed instantly and on demand; they can be configured with the least interaction with the service provider [16].

Mobile computing is a computing paradigm applicable to all programmable, portable, wireless, and conveniently held devices including, but not limited to, smartphones, tablets, pads, smartwatches and laptops that can be used anywhere and at any time. Most e-health applications are intended to be used by healthcare professionals for disease diagnosis, drug reference and medical calculations [17]. Mobiles and especially smartphones are also considered as packages of sensors and hardware features from which context can be extracted.

The continuous up growth of virtualization technology has made Virtual Machines (VM) turn out into a usual way of providing specific tasks which are distinct from the ones performed in the host environment. A VM is the core of the virtualization and it is basically an emulated computer system using specific software and hardware. It aims to allow the users to perform other tasks than the ones restricted by the physical characteristics of a server. A VM keeps going to use the physical system resources (like RAM, CPU, HDD) while it is distinct and apart from the other installed software. Therefore, a VM can be built, transformed, or cancelled with no impact on the host. A VM behaves as the actual hardware and it is perceived as a physical computer. While in the real world a unique application and a single operating system use the physical resources, in a virtual architecture there might be several VMs running simultaneously on a single server through a software layer - hypervisor interposed between the hardware and VM. The VMs can be installed in the cloud server. Among the main advantages of VMs are: a single physical server can host (through hypervisors) multiple VMs with different applications running on, eliminating the need of inefficiently exploited PCs [18]; easy accessibility, scalability, management and maintenance; alternatives for recovery or replacements in case of disasters.

In healthcare VMs have been increasingly applied because of the growing usage of cloud computing in eHealth, of the need for a better management of the health-related data gathered through IoT, of the smart devices and remote monitoring technology

\subsection{Appropriate Smart Services and Applications for Senior Patients}

In healthcare, smart services has the potential to enhance the quality of life in the society. The smart healthcare services for senior patients are part of general IoT services and the protocols required for IoT structure that might suffer small adaptations necessary for the accurate functionality of the healthcare system [19]. Some of such services are briefly presented below:

- Ambient Assisted Living (AAL) comprises all the services that ensure a non-intrusive support for everyday activities taking into consideration the 
particularities of the context and assisted person" [20]. These services enable senior patients to live in their own homes as long as possible (rather than being hospitalized to stationary care facilities, which, in turn, diminish the burden on public healthcare system) and can help them to improve the quality of life, to have an independent life and to keep them safe and happy. Such services, which have to be economically reasonable and user friendly IT-enabled, are used in flexible monitoring of health conditions of elderly individuals and in providing their security. Real time control of the medical status (by using smart services as Glucose level sensing, Electrocardiogram Monitoring, Blood Pressure Monitoring, Body Temperature Monitoring, Oxygen Saturation Monitoring), nutrition tracking and senior behaviour are performed, and the deviations from the normal values are communicated to health professionals for making therapeutic decision making and for taking the necessary actions.

- Internet of m-health things (m-IoT), known as mobile IoT, includes mobile computing, communication technology (in 4G networks) and medical sensors for healthcare services. It is used real-time monitoring system and for messageexchange-based mobility.

- Wearable Device Access - Equipped with WSN, a wearable device can monitor elderly individuals accurately and calculates their glucose level, pulse etc. Smart watches and smart phones are included in wearable devices and can also be used in many healthcare applications;

- Smart Medication Management - The medication management problem causes serious risks to public health and may lead to important financial lost. To overcome these issues, intelligent packaging method for medicine boxes, based on IoT, have been deployed. The ehealth services architecture of tags are used for the medication control system [21];
- Smart Wheelchair Management is a realtime service that introduces full automation for disabled persons. By using various sensors, the smart wheelchair detects and vibrates in unison with the sensation of the disabled person, could monitor the vital signals of the individuals in chair and then stores the data based on user's environments, permitting the location assessment.

\section{Study Case: RO-SmartAgeing Project}

\subsection{Brief presentation of RO-SmartAgeing}

"Non-invasive monitoring and health assessment of the elderly in a smart environment (RO-SmartAgeing)" is an is an ongoing project whose outcomes will be able to support the provision of more advanced elderly-centred healthcare services by facilitating the orientation of medical practice to prevention and personalized assistance.

In this context, it is developed a system for monitoring and assessing the state of health of the elderly, integrating non-invasive motion and physiological sensors for the collection of health parameters, a smart environment with discreet environmental sensors to monitor daily activities and lifestyle and a cloud platform for storing and aggregating data, including the presentation of the results of advanced data analysis.

The smart personalized environment for an elderly patient that will be developed in this project integrates physiological, motion and environmental sensors, interconnected digital devices and technologies capable of providing services and support for elderly well-being, continuous remote monitoring of his/her daily activities, health and safety. The main purpose of the intelligent environment is to allow the elderly person to continue his/her life with dignity in a familiar environment while receiving the best health surveillance, treatment and physical support.

The testbed for monitoring and assessing health status includes:

- A development platform that includes medical devices and eHealth applications (containing biomedical sensors that measure physiological parameters), plus 
the Raspberry Pi (Raspbian Mini System) and the Arduino medical data acquisition board;

- IOT components that include sensors: biomedical (measuring pulse, body temperature, blood oxygen level, blood pressure, etc.), ambient (door, gas, fire, etc.) motion sensors (gyroscope type, accelerometer, depth camera etc.) and portable (smartwatch, GPS patches, etc.). Data collection is continually being aggregated and effectively exploited within the services implemented in the ICIPRO Cloud infrastructure;

- An open-source Thingsboard IoT platform that allows the collection, processing and viewing of data from sensors or other monitoring devices as well as their remote management. The use of Cloud technologies is required for the execution of advanced data analysis algorithms for patient risk assessment or predictions. It will contain:

- The Web service for collecting the online transmitted medical data and for storing them in a NoSQL database;

- The Web interface designed for the visualization of patient medical data collected within a time period;

- A Web application (server) implemented in the Cloud ICIPRO infrastructure that provides services for the client application (that can be a Web application or a mobile / desktop device);
- A client application (for desktop / mobile or web application).

Among the most important functionalities to be developed within the project for the benefit of an elderly patient in the smart environment are:

- Non-invasive monitoring of the current health status in order to define a real elderly profile that presents the current state of some medical parameters (physiological and cognitive);

- Monitoring of current activities and lifestyle;

- Personal safety supervision;

- Defining a model for tracking the locomotor decline and preventing falls;

- Personalized environmental adaptation to allow controlled use of environmental devices or alarm triggering in the event of an abnormal health parameter or an unforeseen and dangerous event;

- Supporting the remote assessment of the elderly's current state of health and early diagnosis by the personal physician;

- Creating facilities to support the social interactions of the elderly person;

- Facilitating the empowerment and involvement of the elderly persons in the optimal management of their own health;

- Identifying the most representative ways of taking care of the elderly.

A special importance is granted to the patientgenerated data that are collected from wearables or other lifestyle and health devices and to their processing and consuming (Figure $1)$. 


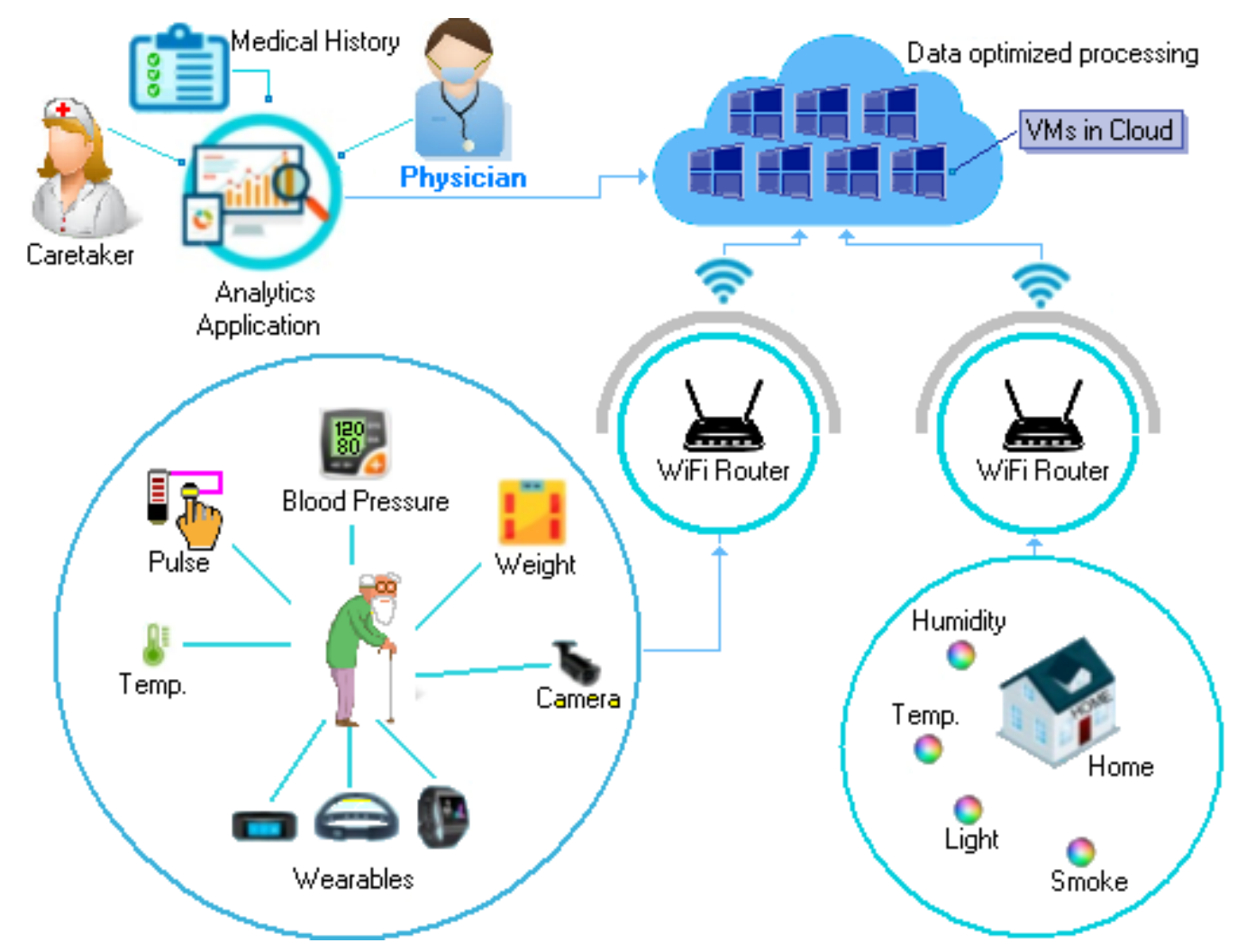

Fig. 1. RO-SmartAgeing: Data collecting, processing and consuming

\subsection{A Proposed Conceptual Solution}

RO-SmartAgeing, as a multi-dimensional remote health Monitoring System based on IoT devices, is confronted with the need for real-time processing of a rapidly increasing volume of data, as physiological and environmental parameters are measured at a high rate (at relatively short intervals of time). In order to monitor a small number of patients, the computing resources provided by a single virtual machine (hosting the Server component and working in the cloud) offer very good satisfaction. Things, however, change dramatically if it is necessary to monitor a high number of patients. In this situation, it becomes mandatory to increase the computing capacity by migrating, on the server, from the "single-virtual machine" to a "multi-virtual machine" system.

Cloud computing is the best method to host the Server component of an eHealth system because it offers:

- Extremely generous and easily configurable storage capacity;
- Possibility of establishing extremely fast communication between computing units (machines).

The conceptual solution that the present paper proposes is mainly concerned with the fructification of very fast physical communication between the computing units. Computing Units are web services running each under its own virtual machine. It must be underlined again that a virtual machine is an operating system that runs under the governing of a host operating system.

RO-SmartAgeing System is intended to manage two types of data streams: the first flow consists of structured medical data provided by the data gathering devices, the second flow consists of information resulting from data processing. Attached to these streams, there are the above-mentioned computing units (Web services), that perform the following types of role:

- Decrypting, decoding and separating the piece of information currently received from the IoT medical device; reencrypting the components thus obtained, followed by their inserting into the 
database as a new record (the useful information will contain the patient's name, the unique identifier, the name of the measured medical parameter, the value of the medical parameter, the time and the day);

- Upon the arrival of a request - decrypting and processing a chosen medical parameter of a certain patient (drawing a graph of the parameter as a function of time, issuing an alarm notification, etc.).

Figure 2 shows the functional scheme for gathering and sending medical parameters. In order to monitor the patient, the patient medical data are collected by specialized IoT sensors and transmitted using wireless network to the cloud resident server.

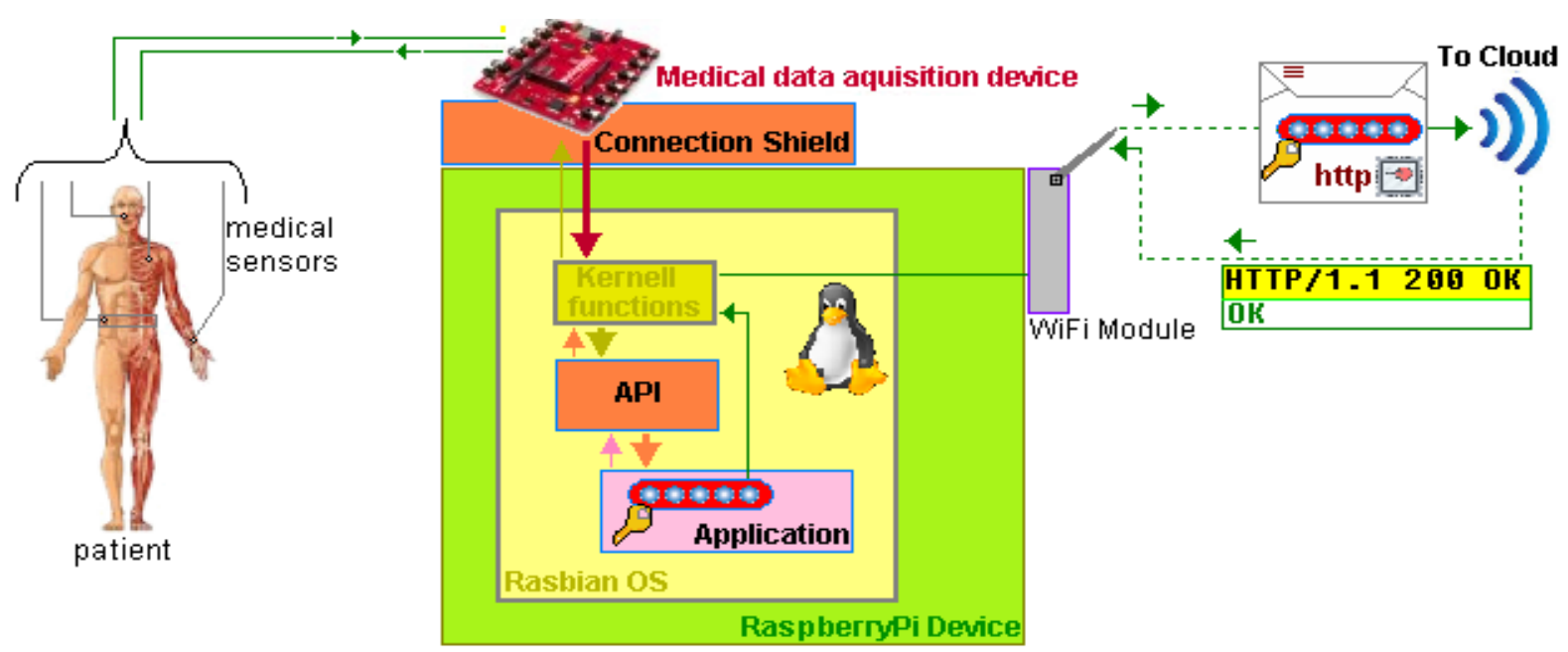

Fig. 2. Gathering and sending medical data to the Cloud resident Server

Two types of sensors: medical (main sensors), "attached" to the patient in order to measure vital parameters, and environmental (secondary sensors), placed in the room where the patient is located. The values of the medical parameters define patient's real-time health status, being immediately packed inside the HTTP-Post request and transmitted to the server-side component of the platform where processing is performed when asked for by remote client application(s).

The proposed conceptual solution aims at optimizing the response time offered by the server component of the system under the constraint of an intensive flow of requests for data processing and real time responses.

In Figure 3 is represented the server-side proposed solution.

The server component (server-side) of the system consists of the following subcomponents:
- (R:1) The service that is intended to receive and organize into the storage space all sent data. It works inside its own virtual machine. The primary role of this service is to insure for the actual data (extracted from the incoming HTTP-Post request) a simultaneous storage in multiple identical databases, each base running in its own virtual machine. The service immediately redirects ("broadcasts") current data to the ensemble of services introduced immediately below. The duplication process of medical data storage has a dual role:

- It allows balancing the processing (computing) effort in order to maintain more or less the same response readiness in the event of a heavy flow of requests issued by various client applications;

- It provides an important safety enhancement for data storage. 


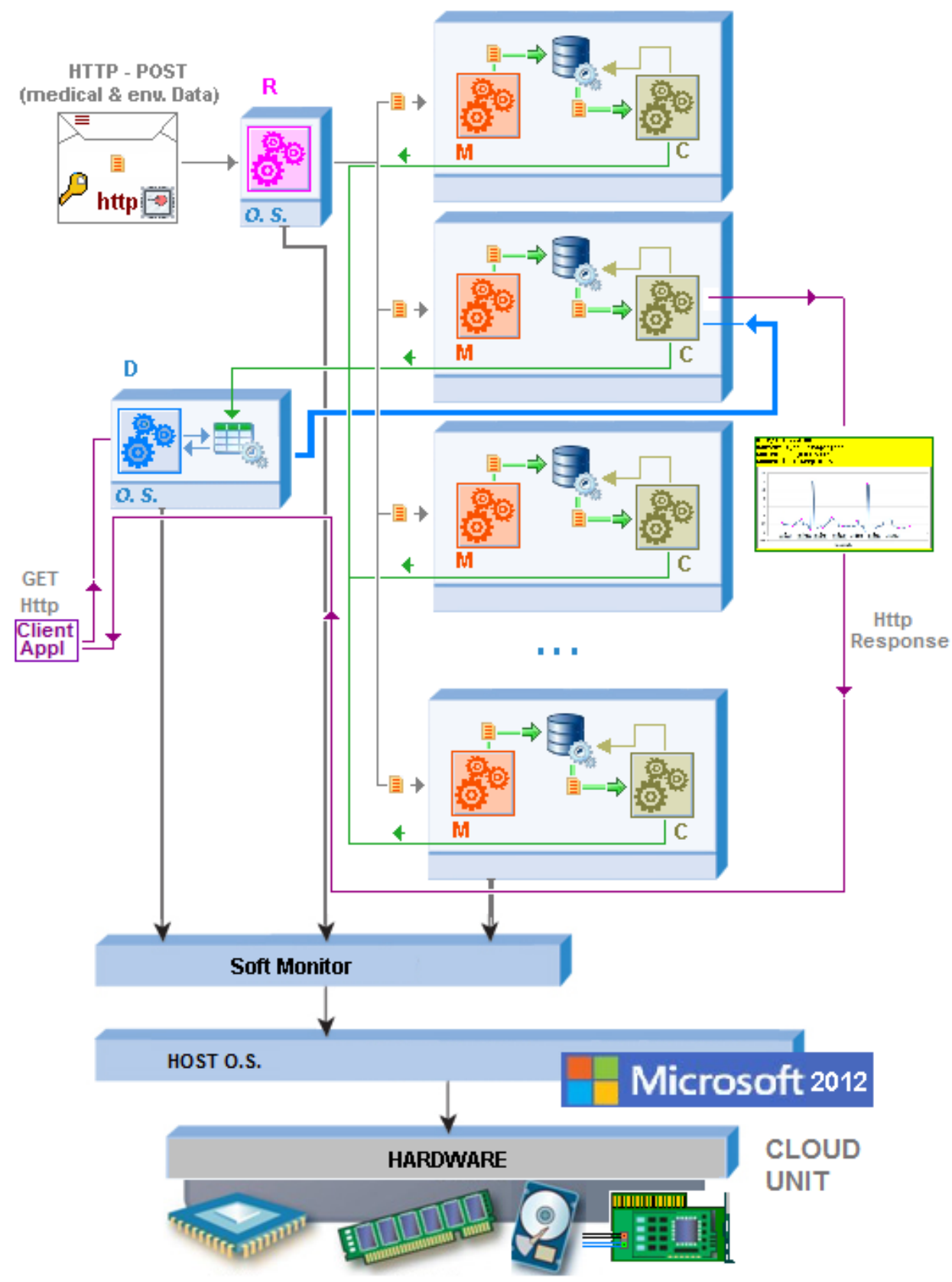

Fig. 3. Optimization of request management based on Services distributed on Multiple VM

- (M:n) Data memorizing (storage) services have the role of writing the data received from the patient in an associated database. Each service works in conjunction with a NoSQL database management system. Each service works inside its own virtual machine.

- (D:1) The dispatch service also works inside its own virtual machine. It captures and manages data processing requests. The role of this service is to assess the distribution of the current computing effort over the actual computing ensemble and to select the most appropriate computing unit (i.e. the most "free") that would be entrusted with resolving the current request. When a request is sent to a certain computing unit, this dispatch service promptly updates to an associated database the loading status of the machine (the computing unit) to which the current request has just been sent.

- (C:n) Services of computational unit type. Each of them serves three main purposes: 
O reads appropriate data (pertaining to the current request) from the associated database.

○ processes retrieved data and send the response to the requesting client application.

$\circ$ instantly after sending the response to the requesting client application, it notifies the dispatcher (through a Http-PUT request) regarding its newly established availability. A single "C" type service and a single "M" type service are hosted by the same Virtual Machine.

It must be stated that every alphanumerical symbol (following the colon, inside de brackets that open each main enumeration of services) indicates the multiplicity of the service(s) within the architecture of the solution (i.e. "1" indicates that a certain service has a single instance, whereas " $n$ " suggests that the introduced service is configured to have multiple instances).

\section{Conclusion}

Monitoring senior persons health in an IoThased smart environment contributes to improving the assessment of physical parameters of the elderly residing not in hospitals, but rather at their own domicile. The amount of healthcare data gathered within this environment is not only huge but also heterogeneous, requiring fast processing. In order for the healthcare data to enjoy efficient storing and processing, server-side applications must be optimized first and foremost.

Integrating IoT and Cloud technologies in the monitoring of physiological and environmental parameters, compounded with developing various predictive models for population health - allow the achievement of innovative personalized assistance services.

In the context of the ongoing ROSmartAgeing project, the authors proposed a server-side architecture aiming at optimizing the response speed (i.e. reducing processing time and shortening waiting intervals) in the most likely scenario of a heavy flow of requests issued by various client applications.
Balancing the computing load is achieved by using an ensemble of dispatching/computing services, each one running under its own virtual machine, the whole mechanism fructifying the extremely high speed at which re-distribution of incoming requests is performed between virtual machines belonging to the same Cloud Unit.

\section{Acknowledgment}

This work was supported from the project "Non-invasive monitoring and health assessment of the elderly in a smart environment (RO-Smart Ageing)" (funded by the Romanian Core Program of the Ministry of Research and Innovation) (2018 -2020).

\section{References}

[1] Eurostat, Population structure and ageing, Available: https://ec. europa.eu/eurostat/statis ticsexplained/index.php?title=Population_str ucture_and_ageing\&oldid=423053\#Past_ and

future_population_ageing_trends_in_the EU, May 2018 April, 2019.

[2] National Institute of Aging, World Health Organization, "Global Health and Aging", October 2011, pp. 8. Available: https://www.nia.nih.gov/research/publicat ion/global-health-and-aging/ humanitysaging.

[3] S. Vitabile, et al., "Medical Data Processing and Analysis for Remote Health and Activities Monitoring", in: Kołodziej J., González-Vélez H. (eds) High-Performance Modelling and Simulation for Big Data Applications. Lecture Notes in Computer Science, vol 11400. Springer, Cham, 2019, pp 186221.

[4] C. Mathias (2017, October), What is virtualization? Way more than just virtual machines, NetworkWorld from IDG [Online].

Available: https://www.networkworld.com/article/3 234795/what-is-virtualization-definitionvirtual-machine-hypervisor.html.

[5] X. Guowen, X. Xiaolong et al.), Fair energy-efficient virtual machine 
scheduling for Internet of Things applications in cloud environment, International Journal of Distributed Sensor Networks, [Online], Volume: 13 issue: 2, 2017.

[6] S. Sharma. (2014). A Review on Efficient Virtual Machine Live Migration: Challenges, requirements and technology of VM migration in cloud, International Journal of Cloud Computing and Services Science (IJ-CLOSER) [Online] 2089$3337 . \quad 3 . \quad 1-8$., Available: https://www.researchgate.net/publication/ 302900831_A_Review_on_Efficient_Vir tual_Machine_Live_Migration_Challeng es_requirements_and_technology_of_V M_migration_in_cloud.

[7] National Institute of Aging, World Health Organization, "Global Health and Aging", October 2011, pp. 8. Available: https://www.nia.nih.gov/research/publicat ion/global-health-and-aging/ humanitysaging.

[8] M. Ianculescu, A. Alexandru and E. Tudora, "Opportunities brought by big data in providing silver digital patients with ICT-based services that support independent living and lifelong learning". In: Proc. 2017 Ninth International Conference on Ubiquitous and Future Networks (ICUFN). IEEE, 2017. pp. 404409.

[9] M. M. Dhanvijay and S. C. Patil, "Internet of Things: A survey of enabling technologies in healthcare and its applications", Computer Networks, 2019.

[10] M. Ebling, "Pervasive Computing and the Internet of Things", In IEEE Pervasive Comput., pp. 2-4, 2016.

[11] M. Kocakulak and I. Butun, "An overview of Wireless Sensor Networks towards internet of things", In Proc. 2017 IEEE 7th Annual Computing and Communication Workshop and Conference (CCWC) 2017 Jan 9, p. 1-6, IEEE. 2017.

[12] J.T. Erber, Ageing and Older Adulthood, third ed. Wiley-Blackwell, Chichester,
2013.

[13] H. Li, J. Wu Y. Gao and Y. Shi, "Examining individuals' adoption of healthcare wearable devices: An empirical study from privacy calculus perspective", International journal of medical informatics, Vol. 88, pp 8-17 Apr 2016.

[14] S.T. Rosenbloom, "Person-generated health and wellness data for health care", J. Am. Med. Inf. Assoc., Vol. 23, Issue 3, pp. 438-439, 2016.

[15] T. Rault, A. Bouabdallah, Y. Challal and F. Marin, "A survey of energy-efficient context recognition systems using wearable sensors for healthcare applications", Pervasive Mob. Comput. Vol. 37, pp. 23-44, 2017.

[16] H. Elazhary, "Internet of Things (IoT), mobile cloud, cloudlet, mobile IoT, IoT cloud, fog, mobile edge, and edge emerging computing paradigms: Disambiguation and research directions", Journal of Network and Computer Applications, 2018.

[17] A. Mosa, I. Yoo and L. Sheets, "A systematic review of healthcare applications for smartphones", BMC Med. Info. Decis. Making, Vol. 12, Issue 67, 2012.

[18] Actus Digital. Virtual Machine or Physical Server. Available: https://actusdigital.com/virtual-machinevs-physical-server/, January 2018 [April, 2019].

[19] M. M. Dhanvijay and S. C. Patil, "Internet of Things: A survey of enabling technologies in healthcare and its applications", Computer Networks, 2019.

[20] H. Steg, H. Strese, C. Loroff, J. Hull, and S. Schmidt,. "Europe is facing a demographic challenge-ambient assisted living offers solutions", IST Project Report on Ambient Assisted Living, 2006.

[21] M. M. Dhanvijay and S. C. Patil, "Internet of Things: A survey of enabling technologies in healthcare and its applications", Computer Networks, 2019. 


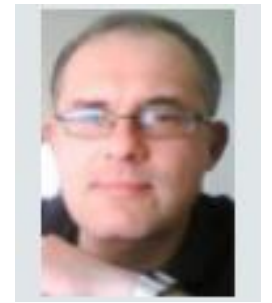

Dragoș NICOLAU is a graduate of the Faculty of Electrical Engineering at the Politehnica University of Bucharest, since 1991. Currently he holds the position of scientific researcher III at ICI, Bucharest. He has skills in developing web, desktop and network applications. He is strongly passionate about the less-explored areas of object-oriented programming. As a field of interest, Dragoș Nicolau also mentions: securing networks, securing JavaScript codes and semiconductor physics. Mr. Nicolau loves to study and deploy software based on execution threads, encryption / compression algorithms, image analysis, and network communications. He has published more than 30 articles in the country and abroad. Between 1997-2002 he performed Academic teaching activity at the Faculty of Electrical Engineering of UPB.

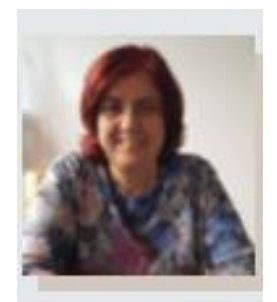

Adriana ALEXANDRU graduated the Faculty of Automatic Control at University Politehnica of Bucharest, the Faculty of Mathematics at University of Bucharest, and the PhD in Applied Informatics at University Politehnica of Bucharest. She is Senior Researcher 1st degree and Professor at Valahia University of Târgoviste. She is General Manager of the National Institute for Research and Development in Informatics, Bucharest She coordinated several national projects in the field of e-Health, climate change, energy efficiency and renewable energies and was scientist in charge of 11 European projects in PECO, INCO COPERNICUS, SAVE, IST, EIE and Comenius European programmes. She is author of 3 books, co-author of 6 books and wrote over 230 scientific papers published in Romania and abroad. Prof. Adriana Alexandru is member of IEEE, VDI, SRIM, WASET, and Evaluation Board for RELANSIN, INFOSOC, CEEX, and PNIII national research programmes and reviewer for FP V, EUREKA, and POC EU programmes and for several journals and conferences. She has been awarded several prizes for excellence in eHealth

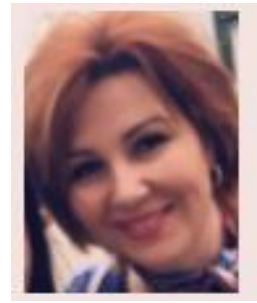

Marilena IANCULESCU graduated the Faculty of Automatic Control and Computer Science, UPB Bucharest. Currently, she is Senior Researcher III and Head of "Society-Oriented IT Systems and Applications" department in National Institute for Research \& Development (ICI), Bucharest, Romania and $\mathrm{PhD}$ student at Computer Science Department, UPB Bucharest. Expertise sector: eHealth \& eInclusion, eLearning, eBusiness, web applications, database systems. She has an extensive experience in project management and software development. She has coordinated and participated in national research projects, mainly in eHealth domain. She was reviewer for several journal and conferences She is author/co-author of more than 100 scientific papers published in Romania and abroad. She has been awarded several prizes for excellence in eHealth. 